\title{
Green Chemical Synthesis and Analgesic Activity of Fluorinated Thiazolidinone, Pyrazolidinone, and Dioxanedione Derivatives
}

\author{
Harshita Sachdeva, ${ }^{1}$ Diksha Dwivedi, ${ }^{1}$ and Pradeep Goyal ${ }^{2}$ \\ ${ }^{1}$ Department of Chemistry, Faculty of Engineering and Technology, Mody Institute of Technology and Science, \\ Lakshmangarh, Sikar, Rajasthan 332311, India \\ ${ }^{2}$ Goenka College of Pharmacy, Goenka Institute of Education and Research (GIER), Lakshmangarh, Sikar, Rajasthan 332311, India
}

Correspondence should be addressed to Harshita Sachdeva; drhmsachdevaster@gmail.com

Received 7 April 2013; Revised 28 June 2013; Accepted 28 June 2013

Academic Editor: William Setzer

Copyright (c) 2013 Harshita Sachdeva et al. This is an open access article distributed under the Creative Commons Attribution License, which permits unrestricted use, distribution, and reproduction in any medium, provided the original work is properly cited.

Facile lemon juice catalyzed green and efficient synthesis of a series of new classes of 5-(fluorinatedbenzylidene)-2-thioxo-1,3-thiazolidin-4-ones (3a-e), 5-methyl-4-(fluorinatedbenzylidene)-2-phenylpyrazolidin-3-ones (5a-e), and 2,2-dimethyl-5(fluorinatedbenzylidene)-1,3-dioxane-4,6-diones (7a-e) by the reaction of fluorinated aromatic aldehydes with active methylene compounds is reported. Lemon juice is natural acid catalyst which is readily available, cheap, nontoxic, and ecofriendly. This method is experimentally simple, clean, high yielding, green, and with reduced reaction times. The product is purified by simple filtration followed by washing with water and drying process. Some of the synthesized compounds have been evaluated "in vivo" for their analgesic activity and all the synthesized compounds are characterized by $\mathrm{IR},{ }^{1} \mathrm{H} \mathrm{NMR},{ }^{13} \mathrm{C} \mathrm{NMR},{ }^{19} \mathrm{~F}$ NMR, and mass spectral studies.

\section{Introduction}

The steady growth of interest in the synthesis of heterocyclic compounds is connected with their raised biological activity and also with the fact that these compounds make possible the development of novel materials of unique properties. Pyrazolone is a biologically important scaffold associated with multiple pharmacological activities such as antimicrobial [1], anti-inflammatory [2], analgesic [3], antidepressant [4], anticonvulsant [5], antidiabetic [6], antihyperlipidemic [7], antiviral [8], antitubercular [9], antioxidant [10], and anticancer activites $[11,12]$. The synthesis of pyrazolone and its derivatives has engrossed substantial attention from organic and medicinal chemists for many years as they belong to a class of compounds with proven utility in medicinal chemistry.

One very interesting and promising class of heterocycles is the 4-thiazolidinone ring system. It represents a class of chemical products with interesting pharmacological and biological activities [13-18] including antidiabetic, antitubercular, anti-HIV, antiparasitic, hypnotic, and anathematic agents.
Furthermore, the reactivity of the Meldrum's acid (2,2-dimethyl-1,3-dioxan-4,6-dione) as a methylene active compound was explored about 40 years after its preparation, when the structure was correctly attributed by Davidson and Bernhard [19] assigning the acidic proton to the central carbon, and its high acidity is still object of study [20]. It is known that the Meldrum's acid undergoes standard Knoevenagel condensation with aromatic and heteroaromatic aldehydes furnishing the corresponding arylidene derivatives, which are versatile substrates for different kinds of reactions [21, 22]. They are useful intermediates for cycloaddition reaction and for the synthesis of heterocyclic compounds with potential pharmacological activity [23].

Several methods have been developed for the preparation of thiazolidinone, pyrazolone, and dioxanedione derivatives. The most common is Knoevenagel condensation between aromatic aldehydes and various active methylene compounds carried out in glacial acetic acid containing anhydrous sodium acetate [24]. Instead of sodium acetate, acetic anhydride [25], ethanolamine [26], and ammonium chloride in ammonia [27-29] have also been used as catalysts. Other 
<smiles>CC1C(N2Cc3ccccc3C2=O)c2cc(OC(F)(F)F)ccc2OC1(C)C</smiles>

Celikalim<smiles>Nc1nc2ccc(OC(F)(F)F)cc2s1</smiles>

FIgURE 1: Fluorine containing drugs.

derivatives were also prepared using glycine and sodium carbonate as catalysts, ionic liquid, and acidic alumina as solid support, borate zirconia [30-34].

More recent methods for the preparation of 5-benzylidene-2-thioxothiazolidin-4-ones, 4-arylidene-3-methyl1-phenyl-5-pyrazolone derivatives, and 5-benzylidene-2,2dimethyl-1,3-dioxane-4,6-dione have been reported which involve Knoevenagel condensation of aromatic aldehydes with 2-thioxothiazolidin-4-one/3-methyl-1-phenyl-1H-pyrazol-5(4H)-one/Meldrum acid catalyzed by a basic functionalized ionic liquid [35], nanoparticles [36, 37], triphenylphosphine [38], and 1-butyl-3-methylimidazolium hydroxide ([bmim] $[\mathrm{OH}])[39,40]$. Knoevenagel condensation is also reported under microwave irradiation for the synthesis of 5arylidene-4-thiazolidinones [41]. These derivatives are also synthesized using tetrabutylammonium bromide (TBAB) as a phase transfer catalyst in water under microwave irradiation [42]. However, in spite of their utility, some methods suffer from disadvantages like long reaction times, low yields, chemical hazards, and environmental pollution.

In recent years, organic research is mainly focused on the development of green methods to synthesize various organic compounds through the use of alternative green catalyst to replace hazardous strong acidic or basic catalyst commonly used in organic synthesis. Nowadays, many organic transformations have been carried out using biocatalysts or intact plant systems. Recently, use of lemon juice as natural catalyst is reported [43] in few chemical reactions because it is inexpensive, most abundant in nature, nonhazardous, and ecofriendly. It exhibits unique reactivity and selectivity. As lemon juice is acidic in nature $(\mathrm{pH} \approx 2-3)$ and percentage of citric acid $(5 \%-7 \%)$ is more than other acids, it works as acid catalyst.

In addition, incorporation of fluorine further enhances the biological activity by increasing solubility in lipoid material and fat deposits in the body. Compounds of medicinal interest containing trifluoromethyl substituents, including anaesthetics, antipsychotics, antibiotics and a few antimalarials were reviewed in 1958 [44]. Antibiotic multi drug resistance is a major and continuing public health concern and some clinicians are switching to replacements such as the fluoroquinolones [45]. A recent review [46] has highlighted pesticides containing the $\mathrm{CF}_{3} \mathrm{O}$ group, and its authors have argued that a $\mathrm{CF}_{3} \mathrm{O}$ substituent can advantageously replace a fluorine atom in most molecules with the benefit of increased lipid solubility. Many drugs with enhanced effectiveness and selectivity contain the $\mathrm{CF}_{3} \mathrm{O}$ moiety (e.g., celikalim, roflumilast and riluzole) (Figure 1).

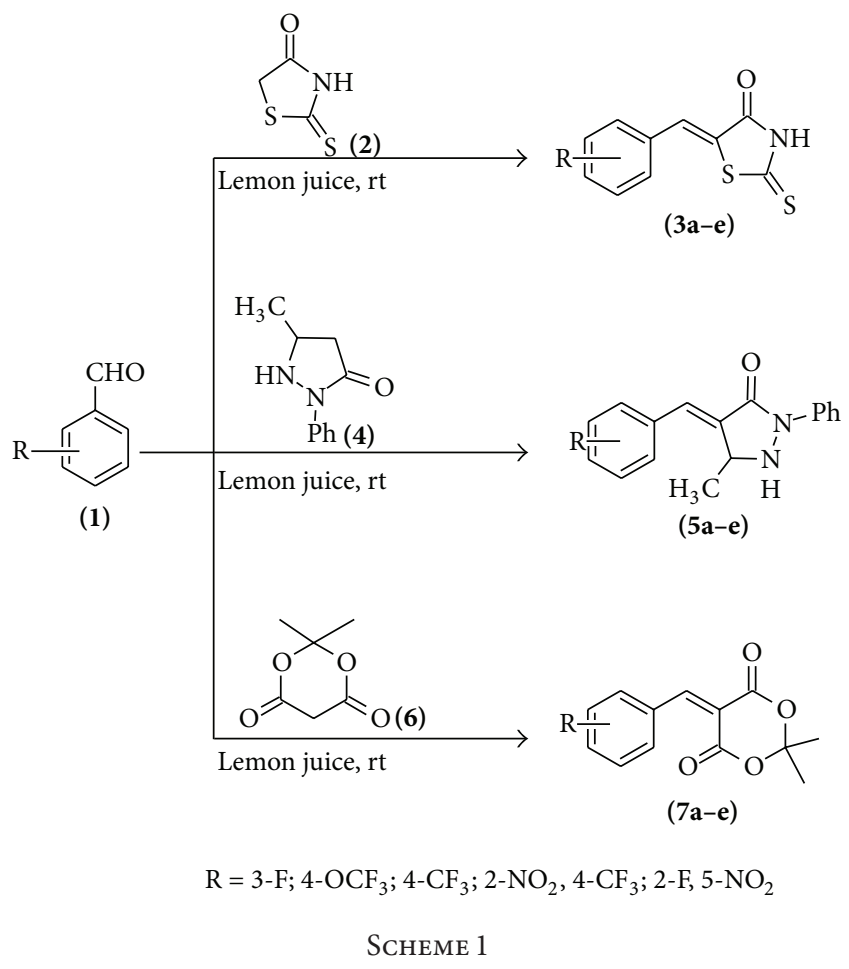

In Scheme 1, we have synthesized for the first time a series of new class of fluorine containing olefinic compounds from Knoevenagel condensation of fluoro-substituted aromatic aldehydes (1) with active methylene compounds, 2thioxo-4-thiazolidinone (2)/3-methyl-1-phenyl-2-pyrazolin5-one (4)/Meldrum's acid (6) in the presence of lemon juice as natural acid catalyst at room temperature. The compounds synthesized by various methods have been characterized by their melting points, elemental analyses, IR, ${ }^{1} \mathrm{HNMR}$, ${ }^{13} \mathrm{CNMR},{ }^{19} \mathrm{FNMR}$, and mass spectral studies.

\section{Results and Discussion}

In continuation to our interest on environmentally benign synthesis of heterocyclic compounds [47-50], we now report the green synthesis of fluorinated thiazolidinone (3a-e), pyrazolidinone $(\mathbf{5} \mathbf{a}-\mathbf{e})$ and dioxanedione derivatives $(7 \mathbf{a}-\mathbf{e})$ via Knoevenagel condensation at room temperature in the presence of catalytic amount of lemon juice as natural acid catalyst (Scheme 1). We have extensively studied the title reaction taking two parameters, namely, type of catalysts and type of solvent. In order to optimize the reaction conditions, 
TABLE 1: Synthesis of 5-benzylidene-2-thioxo-1,3-thiazolidin-4-one 3a under different catalysts.

\begin{tabular}{lcccc}
\hline Entry & Catalyst & $\begin{array}{c}\text { Solvent/temperature } \\
\left({ }^{\circ} \mathrm{C}\right)\end{array}$ & $\begin{array}{c}\text { Time } \\
(\mathrm{hrs})\end{array}$ & $\begin{array}{c}\text { Yield } \\
(\%)\end{array}$ \\
\hline 1 & Boric acid & $\mathrm{H}_{2} \mathrm{O} / 80$ & 5 & 67 \\
2 & Oxalic acid & $\mathrm{H}_{2} \mathrm{O} / 80$ & 5 & 72 \\
3 & Alum & $\mathrm{H}_{2} \mathrm{O} / 80$ & 3 & 82 \\
4 & Lemon juice & $\mathrm{H}_{2} \mathrm{O} / 80$ & 3 & 84 \\
\hline
\end{tabular}

the synthesis of compound $\mathbf{3 a}$ was used as a model reaction and a mixture of 3-fluoro benzaldehyde and 2-thioxo-4thiazolidinone was magnetically stirred in presence of various catalysts as shown in Table 1 . When reaction was carried out in aqueous medium in the presence of oxalic acid, boric acid, alum, and lemon juice (Table 1, entry 1-4), lemon juice provided the best yield as compared to other catalysts.

Furthermore, we studied the role of solvent on the synthesis of title compounds and found that the solvent played a crucial role in this reaction (Table 2, entry 1, 2, 3). Ethanol, methanol, dichloromethane were all able to facilitate but it took longer time (4-5 hrs) to complete the reaction with low yield $(66-75 \%)$ of the product. We extended our studies and carried out the reaction in the absence of any solvent and presence of lemon juice at room temperature, $92 \%$ yield of the product was obtained in $1.5 \mathrm{hrs}$. (Table 2, entry 4 ). With these optimal conditions in hand, we examined the scope of this Knoevenagel condensation reaction. Results indicate that lemon juice is the best catalyst at room temperature for the synthesis of olefinic compounds (Table 3). As lemon juice is acidic in nature $(\mathrm{pH} \approx 2-3)$ and percentage of citric acid (5\%$7 \%)$ is more than other acids, it works as acid catalyst for the synthesis of fluorinated thiazolidinone (3a-e), pyrazolidinone (5a-e), and dioxanedione derivatives (7a-e). Using this methodology, these reactions were completed in shorter reaction times (1-2 hrs) at room temperature $\left(25^{\circ} \mathrm{C}\right)$ with yields of the product ranging from $90 \%$ to $95 \%$. For the Knoevenagel condensation reaction, we have used extract of Citrus limonum species of lemon as natural catalyst for synthesis of arylidenes. To our satisfaction, we found that the use of $2 \mathrm{~mL}$ of lemon juice resulted in quantitative yield (90\%-95\%) of the corresponding olefinic compounds within 1 to $2 \mathrm{hrs}$. The purity of the compounds was checked by TLC using silica gel$\mathrm{G}$ as adsorbent. We have also carried out this reaction using citric acid separately; reaction took place successfully as observed on TLC.

The product is isolated in pure form and does not require further purification and crystallization. Hence, other compounds were also synthesized at room temperature following the similar procedure. The chemical structures all the synthesized compounds have been confirmed by IR, ${ }^{1} \mathrm{HNMR}$, ${ }^{19} \mathrm{FNMR},{ }^{13} \mathrm{CNMR}$, and mass spectral studies.

The IR spectra of $\mathbf{3 a - e}$ showed absorption bands at $3320-3350 \mathrm{~cm}^{-1}$ due to $\mathrm{NH}$ stretching of amide, $1680-$ $1692 \mathrm{~cm}^{-1}$ due to $\mathrm{C}=\mathrm{O}, 1578-1596 \mathrm{~cm}^{-1}$ due to $\mathrm{C}=\mathrm{C}$, and $1131-$ $1211 \mathrm{~cm}^{-1}$ due to $C=S$ Stretching, which confirms with the formation of compounds $\mathbf{3} \mathbf{a}-\mathbf{e}$. The ${ }^{1}$ HNMR spectrum of $\mathbf{3 b}$
TABLE 2: Synthesis of 5-benzylidene-2-thioxo-1,3-thiazolidin-4-one (3a) under different solvents.

\begin{tabular}{lcccc}
\hline Entry & Catalyst & $\begin{array}{c}\text { Solvent/temperature } \\
\left({ }^{\circ} \mathrm{C}\right)\end{array}$ & $\begin{array}{c}\text { Time } \\
(\mathrm{hrs})\end{array}$ & $\begin{array}{c}\text { Yield } \\
(\%)\end{array}$ \\
\hline 1 & Lemon juice & $\mathrm{EtOH} / 78$ & 4 & 75 \\
2 & Lemon juice & $\mathrm{CH}_{3} \mathrm{OH} / 65$ & 4 & 72 \\
3 & Lemon juice & $\mathrm{CH}_{2} \mathrm{Cl}_{2} / 40$ & 5 & 66 \\
4 & Lemon juice & Room temperature & 1.5 & 92 \\
\hline
\end{tabular}

showed peaks at $\delta 8.65(\mathrm{~s}, 1 \mathrm{H}, \mathrm{NH}), 8.02(\mathrm{~s}, 1 \mathrm{H}, \mathrm{CH})$, and 6.79-7.98 (m, 4H, Ar-H) ppm. Formation of compound $\mathbf{3 b}$ was further confirmed on the basis of ${ }^{13} \mathrm{CNMR}$ spectrum. In the ${ }^{13} \mathrm{CNMR}$ spectrum, sharp signals were observed at $\delta 200$ $(\mathrm{C}=\mathrm{S}), 168.37$ (C=O), 161.23 (C-O), $143(\mathrm{CH}), 120.48$ (C=C, aliphatic carbon), $122\left(\mathrm{OCF}_{3}\right)$, and 138.25-119.36 (aromatic carbons) ppm. Mass spectrum of compound $\mathbf{3 b}$ showed molecular ion peak $\left[\mathrm{M}^{+}+1\right]$ at $306 \mathrm{~m} / \mathrm{z}(54 \%)$ corresponding to its molecular weight along with base peak observed at $\mathrm{m} / \mathrm{z}$ $122(100 \%)$ and other relevant peaks were observed at m/z 246 (39\%), 236 (84\%), 220 (70\%), 95 (15\%), and 79 (58\%).

The IR spectra of $\mathbf{5 a - e}$ showed absorption bands at 2915$3129 \mathrm{~cm}^{-1}$ due to $\mathrm{CH}$ str of methine, $1670-1688 \mathrm{~cm}^{-1}$ due to $\mathrm{C}=\mathrm{O}$ str, and $1586-1591 \mathrm{~cm}^{-1}$ due to $\mathrm{C}=\mathrm{C}$, which confirms with the formation of compounds $5 \mathbf{a}-\mathbf{e}$. The ${ }^{1} \mathrm{HNMR}$ spectrum of $5 \mathbf{d}$ showed peaks at $\delta 7.73(\mathrm{~s}, 1 \mathrm{H}, \mathrm{CH}), 7.19-7.71$ (comp, 8H, Ar-H), 3.51 (s, 1H, CH), 2.52 (s, 1H, NH), and 1.24 $\left(\mathrm{s}, 3 \mathrm{H}, \mathrm{CH}_{3}\right)$ ppm. Formation of compound $\mathbf{5 d}$ was further confirmed on the basis of ${ }^{13} \mathrm{CNMR}$ spectrum. In the ${ }^{13} \mathrm{CNMR}$ spectrum, sharp signals were observed at $\delta 162.50(\mathrm{C}=\mathrm{O})$, $134.82(\mathrm{CH}), 130.82\left(\mathrm{C}=\mathrm{C}\right.$, aliphatic carbon), $123.13\left(\mathrm{CF}_{3}\right)$, 139.82-112.12 (aromatic carbons), and $21.73\left(\mathrm{CH}_{3}\right) \mathrm{ppm}$.

The IR spectra of 7a-e showed absorption bands at 2915$3083 \mathrm{~cm}^{-1}$ due to $\mathrm{CH}$ str of methine, $1630-1685 \mathrm{~cm}^{-1}$ due to $\mathrm{C}=\mathrm{O}$ str, $1554-1590 \mathrm{~cm}^{-1}$ due to $\mathrm{C}=\mathrm{C}$, and $1055-1145 \mathrm{~cm}^{-1}(\mathrm{C}-$ $\mathrm{O})$ which confirms with the formation of compounds. The ${ }^{1} \mathrm{HNMR}$ spectrum of $7 \mathbf{a}$ showed peaks at $\delta 8.97(\mathrm{~s}, 1 \mathrm{H}, \mathrm{CH})$, 7.70-7.84 (m, 4H, Ar-H), and $2.12\left(\mathrm{~s}, 6 \mathrm{H}, \mathrm{CH}_{3}\right)$ ppm. Formation of compound $7 \mathbf{a}$ was further confirmed on the basis of ${ }^{13} \mathrm{CNMR}$ spectrum. In the ${ }^{13} \mathrm{CNMR}$ spectrum, sharp signals were observed at $\delta 164.91(\mathrm{C}=\mathrm{O}), 160.42(\mathrm{C}-\mathrm{F}), 154.08$ (CH), 123.74 ( $\mathrm{C}=\mathrm{C}$, aliphatic carbon), 134.02-118.12 (aromatic carbons), $105.18(\mathrm{O}-\mathrm{C}-\mathrm{O})$, and $26.16\left(\mathrm{CH}_{3}\right) \mathrm{ppm}$. The mass spectrum of compound 7 a showed molecular ion peak $\left[\mathrm{M}^{+}\right]$ at $250 \mathrm{~m} / \mathrm{z}(30 \%)$ corresponding to its molecular weight along with base peak observed at m/z $176(100 \%)$ and other relevant peaks were observed at m/z 123 (13\%), 100 (34\%), and 63 (9\%). Spectral analyses of all the synthesized compounds are given in Tables 4, 5, and 6.

\section{Experimental}

General. Reagents and solvents were obtained from commercial sources and used without further purification. Melting points were determined on a Toshniwal apparatus. The ${ }^{1} \mathrm{H}$ NMR and ${ }^{13} \mathrm{C}$ NMR of synthesized compounds have been carried out at SAIF, Punjab University, Chandigarh, India. 
TABLE 3: Experimental and analytical data of 5-(fluorinatedbenzylidene)-2-thioxo-1,3-thiazolidin-4-ones (3a-e), 5-methyl-4-(fluorinatedbenzylidene)-2-phenylpyrazolidin-3-ones (5a-e), and 2,2-dimethyl-5-(fluorinatedbenzylidene)-1,3-dioxane-4,6-diones (7a-e).

\begin{tabular}{|c|c|c|c|c|c|c|c|}
\hline \multirow{2}{*}{ Entry } & \multirow{2}{*}{$\mathrm{R}$} & \multirow{2}{*}{ Time (hrs/min) } & \multirow{2}{*}{ Yield (\%) } & \multirow{2}{*}{ M.P. $\left({ }^{\circ} \mathrm{C}\right)$} & \multicolumn{3}{|c|}{ Analysis calcd. (found) (\%) } \\
\hline & & & & & $\mathrm{C}$ & $\mathrm{H}$ & $\mathrm{N}$ \\
\hline $3 \mathbf{a}$ & $3-\mathrm{F}$ & $1.5 \mathrm{hrs}$ & 95 & 132 & $50.19(50.04)$ & $2.53(2.50)$ & $5.85(5.88)$ \\
\hline $3 \mathbf{b}$ & $4-\mathrm{OCF}_{3}$ & $1 \mathrm{hrs}$ & 90 & 115 & $43.28(43.08)$ & $1.98(1.95)$ & $4.59(4.61)$ \\
\hline $3 c$ & $4-\mathrm{CF}_{3}$ & $70 \mathrm{~min}$ & 92 & 172 & $45.67(45.35)$ & $2.09(2.07)$ & $4.84(4.82)$ \\
\hline $3 d$ & $2-\mathrm{NO}_{2}, 4-\mathrm{CF}_{3}$ & $100 \mathrm{~min}$ & 91 & 110 & $39.52(39.75)$ & $1.51(1.53)$ & $8.38(8.35)$ \\
\hline $3 e$ & $2-\mathrm{F}, 5-\mathrm{NO}_{2}$ & $2 \mathrm{hrs}$ & 94 & 89 & $42.25(42.05)$ & $1.77(1.75)$ & $9.85(9.81)$ \\
\hline $5 \mathbf{a}$ & $3-\mathrm{F}$ & $40 \mathrm{~min}$ & 90 & 102 & $72.32(72.15)$ & $5.36(5.33)$ & $9.92(9.95)$ \\
\hline $5 \mathbf{b}$ & $4-\mathrm{OCF}_{3}$ & $50 \mathrm{~min}$ & 93 & 85 & $62.07(62.25)$ & $4.34(4.36)$ & $8.04(8.00)$ \\
\hline $5 c$ & $4-\mathrm{CF}_{3}$ & $1 \mathrm{hrs}$ & 94 & 105 & $65.06(65.25)$ & $4.55(4.52)$ & $8.43(8.40)$ \\
\hline $5 d$ & $2-\mathrm{NO}_{2}, 4-\mathrm{CF}_{3}$ & $60 \mathrm{~min}$ & 90 & 162 & $57.30(57.08)$ & $3.74(3.72)$ & 11.14 \\
\hline $5 e$ & $2-\mathrm{F}, 5-\mathrm{NO}_{2}$ & $70 \mathrm{~min}$ & 93 & 180 & $62.38(62.20)$ & $4.31(4.34)$ & $12.84(12.82)$ \\
\hline $7 \mathbf{a}$ & $3-\mathrm{F}$ & $1.5 \mathrm{hrs}$ & 91 & 104 & $62.40(62.56)$ & $4.43(4.45)$ & - \\
\hline $7 \mathbf{b}$ & $4-\mathrm{OCF}_{3}$ & $2 \mathrm{hrs}$ & 90 & 143 & $53.17(53.34)$ & $3.51(3.49)$ & - \\
\hline $7 \mathrm{c}$ & $4-\mathrm{CF}_{3}$ & $2 \mathrm{hrs}$ & 95 & 164 & $56.01(56.22)$ & $3.69(3.67)$ & - \\
\hline $7 d$ & $2-\mathrm{NO}_{2}, 4-\mathrm{CF}_{3}$ & $2 \mathrm{hrs}$ & 92 & 110 & $48.71(48.51)$ & $2.92(2.94)$ & $4.06(4.03)$ \\
\hline $7 e$ & 2-F, 5- $\mathrm{NO}_{2}$ & $160 \mathrm{~min}$ & 94 & 115 & $52.89(52.69)$ & $3.41(3.39)$ & $4.74(4.71)$ \\
\hline
\end{tabular}

TABLE 4: Spectral data of 5-(fluorinatedbenzylidene)-2-thioxo-1,3-thiazolidin-4-ones (3a-e).

\begin{tabular}{|c|c|c|c|}
\hline Entry & $\operatorname{IR}\left(\mathrm{cm}^{-1}\right)$ & ${ }^{1} \mathrm{H}$ NMR $(\delta \mathrm{ppm})$ & ${ }^{13} \mathrm{C}$ NMR $(\delta \mathrm{ppm})$ \\
\hline $3 \mathbf{a}$ & $\begin{array}{c}3350(\mathrm{NH} \text { str of amide }), 1590 \\
(\mathrm{C}=\mathrm{C}), 1131(\mathrm{C}=\mathrm{S} \text { str }), 1680(\mathrm{C}=\mathrm{O})\end{array}$ & $\begin{array}{c}8.01(\mathrm{~s}, 1 \mathrm{H}, \mathrm{NH}), 7.42(\mathrm{~s}, 1 \mathrm{H}, \mathrm{CH}) \\
7.14(\mathrm{~d}, 2 \mathrm{H}, \mathrm{Ar}-\mathrm{H}, J=8.2 \mathrm{~Hz}), 7.30 \\
\quad(\mathrm{~d}, 2 \mathrm{H}, \mathrm{Ar}-\mathrm{H}, J=8.2 \mathrm{~Hz})\end{array}$ & $\begin{array}{l}200(\mathrm{C}=\mathrm{S}), 168.31(\mathrm{C}=\mathrm{O}), 142.33(\mathrm{CH}), 120.48(\mathrm{C}=\mathrm{C} \text {, } \\
\text { aliphatic carbon), 138.25-119.36 (aromatic carbons) }\end{array}$ \\
\hline $3 b$ & $\begin{array}{c}3357 \text { (NH str of amide), } 1596(\mathrm{C}=\mathrm{C}) \\
1211(\mathrm{C}=\mathrm{S} \text { str }), 1682(\mathrm{C}=\mathrm{O})\end{array}$ & $\begin{array}{c}8.65(\mathrm{~s}, 1 \mathrm{H}, \mathrm{NH}), 8.02(\mathrm{~s}, 1 \mathrm{H}, \mathrm{CH}) \\
7.98(\mathrm{~d}, 2 \mathrm{H}, \mathrm{Ar}-\mathrm{H}, J=8.0 \mathrm{~Hz}), 6.79 \\
(\mathrm{~d}, 2 \mathrm{H}, \mathrm{Ar}-\mathrm{H}, J=8.0 \mathrm{~Hz})\end{array}$ & $\begin{array}{c}200(\mathrm{C}=\mathrm{S}), 168.37(\mathrm{C}=\mathrm{O}), 161.23(\mathrm{C}-\mathrm{O}) 143(\mathrm{CH}), \\
120.48\left(\mathrm{C}=\mathrm{C}, \text { aliphatic carbon), } 122\left(\mathrm{CF}_{3}\right), 138.25-119.36\right. \\
\text { (aromatic carbons) }\end{array}$ \\
\hline $3 c$ & $\begin{array}{c}3348(\mathrm{NH} \text { str of amide }), 1578 \\
(\mathrm{C}=\mathrm{C}), 1168(\mathrm{C}=\mathrm{S} \text { str }), 1686(\mathrm{C}=\mathrm{O})\end{array}$ & $\begin{array}{c}8.32(\mathrm{~s}, 1 \mathrm{H}, \mathrm{NH}), 7.50(\mathrm{~s}, 1 \mathrm{H}, \mathrm{CH}) \\
7.46(\mathrm{~d}, 2 \mathrm{H}, \mathrm{Ar}-\mathrm{H}, J=8.5 \mathrm{~Hz}), 7.32 \\
(\mathrm{~d}, 2 \mathrm{H}, \mathrm{Ar}-\mathrm{H}, J=8.5 \mathrm{~Hz})\end{array}$ & $\begin{array}{c}198.55(\mathrm{C}=\mathrm{S}), 169.30(\mathrm{C}=\mathrm{O}), 143(\mathrm{CH}), 120.48(\mathrm{C}=\mathrm{C} \\
\text { aliphatic carbon), } 122.06\left(\mathrm{CF}_{3}\right), 138.25-119.92 \text { (aromatic } \\
\text { carbons) }\end{array}$ \\
\hline $3 d$ & $\begin{array}{c}3320 \text { (NH str of amide), } 1590 \\
(\mathrm{C}=\mathrm{C}), 1136(\mathrm{C}=\mathrm{S} \text { str }), 1688(\mathrm{C}=\mathrm{O})\end{array}$ & $\begin{array}{c}8.62(\mathrm{~s}, 1 \mathrm{H}, \mathrm{NH}), 7.50(\mathrm{~s}, 1 \mathrm{H}, \mathrm{CH}) \\
7.43(\mathrm{~s}, 1 \mathrm{H}, \mathrm{Ar}-\mathrm{H}), 7.26(\mathrm{~d}, 2 \mathrm{H}, \mathrm{Ar}-\mathrm{H}, \\
J=8.2 \mathrm{~Hz})\end{array}$ & $\begin{array}{c}201(\mathrm{C}=\mathrm{S}), 168.32(\mathrm{C}=\mathrm{O}), 143.44(\mathrm{CH}), 121.17(\mathrm{C}=\mathrm{C}, \\
\text { aliphatic carbon), } 118.56\left(\mathrm{CF}_{3}\right), 146.25-120.36 \text { (aromatic } \\
\text { carbons) }\end{array}$ \\
\hline $3 e$ & $\begin{array}{c}3342(\mathrm{NH} \text { str of amide }), 1584 \\
(\mathrm{C}=\mathrm{C}), 1161(\mathrm{C}=\mathrm{S} \text { str }), 1692(\mathrm{C}=\mathrm{O})\end{array}$ & $\begin{array}{l}8.51(\mathrm{~s}, 1 \mathrm{H}, \mathrm{NH}), 7.44(\mathrm{~s}, 1 \mathrm{H}, \mathrm{Ar}-\mathrm{H}) \\
7.28(\mathrm{~d}, 2 \mathrm{H}, \mathrm{Ar}-\mathrm{H}, J=8.0 \mathrm{~Hz})\end{array}$ & $\begin{array}{c}200(\mathrm{C}=\mathrm{S}), 168.37(\mathrm{C}=\mathrm{O}), 161.03(\mathrm{C}-\mathrm{F}), 143(\mathrm{CH}), \\
120.48(\mathrm{C}=\mathrm{C} \text {, aliphatic carbon), } 138.25-119.36(\text { aromatic } \\
\text { carbons) }\end{array}$ \\
\hline
\end{tabular}

TABLE 5: Spectral data of 5-methyl-4-(fluorinatedbenzylidene)-2-phenylpyrazolidin-3-ones (5a-e).

\begin{tabular}{|c|c|c|c|}
\hline Entry & $\operatorname{IR}\left(\mathrm{cm}^{-1}\right)$ & ${ }^{1} \mathrm{H}$ NMR $(\delta)$ & ${ }^{13} \mathrm{C}$ NMR $(\delta)$ \\
\hline $5 a$ & $\begin{array}{c}3129(\mathrm{CH}), 1670 \\
(\mathrm{C}=\mathrm{O}), 1586(\mathrm{C}=\mathrm{C})\end{array}$ & $\begin{array}{c}7.42(\mathrm{~s}, 1 \mathrm{H}, \mathrm{CH}), 7.14-7.30(\mathrm{comp}, 9 \mathrm{H}, \mathrm{Ar}-\mathrm{H}) \\
3.48(\mathrm{~s}, 1 \mathrm{H}, \mathrm{CH}), 2.01(\mathrm{~s}, 1 \mathrm{H}, \mathrm{NH}), 1.20(\mathrm{~s} \\
\left.3 \mathrm{H}, \mathrm{CH}_{3}\right)\end{array}$ & $\begin{array}{c}163.17(\mathrm{C}-\mathrm{F}), 161.31(\mathrm{C}=\mathrm{O}), 135.33(\mathrm{CH}), 130.48(\mathrm{C}=\mathrm{C}, \\
\text { aliphatic carbon), } 142.05-115.16 \text { (aromatic carbons), } \\
24.13\left(\mathrm{CH}_{3}\right)\end{array}$ \\
\hline $5 \mathbf{b}$ & $\begin{array}{c}3073(\mathrm{CH}), 1677 \\
(\mathrm{C}=\mathrm{O}), 1586(\mathrm{C}=\mathrm{C})\end{array}$ & $\begin{array}{c}7.67(\mathrm{~s}, 1 \mathrm{H}, \mathrm{CH}), 7.38-7.20(\mathrm{comp}, 9 \mathrm{H} \\
\mathrm{Ar}-\mathrm{H}), 3.46(\mathrm{~s}, 1 \mathrm{H}, \mathrm{CH}), 2.11(\mathrm{~s}, 1 \mathrm{H}, \mathrm{NH}) \\
1.23\left(\mathrm{~s}, 3 \mathrm{H}, \mathrm{CH}_{3}\right)\end{array}$ & $\begin{array}{c}162.17(\mathrm{C}-\mathrm{O}), 161.40(\mathrm{C}=\mathrm{O}), 133.68(\mathrm{CH}), 130.83(\mathrm{C}=\mathrm{C}, \\
\text { aliphatic carbon), } 122\left(\mathrm{CF}_{3}\right), 141.85-114.32(\text { aromatic } \\
\text { carbons), } 23.93\left(\mathrm{CH}_{3}\right)\end{array}$ \\
\hline $5 c$ & $\begin{array}{c}2915(\mathrm{CH}), 1675 \\
(\mathrm{C}=\mathrm{O}), 1589(\mathrm{C}=\mathrm{C})\end{array}$ & $\begin{array}{c}7.50(\mathrm{~s}, 1 \mathrm{H}, \mathrm{CH}), 7.34-7.17(\mathrm{comp}, 9 \mathrm{H}, \mathrm{Ar}-\mathrm{H}) \\
3.48(\mathrm{~s}, 1 \mathrm{H}, \mathrm{CH}), 2.08(\mathrm{~s}, 1 \mathrm{H}, \mathrm{NH}), 1.53(\mathrm{~s} \\
\left.3 \mathrm{H}, \mathrm{CH}_{3}\right)\end{array}$ & $\begin{array}{c}161.78(\mathrm{C}=\mathrm{O}), 135.21(\mathrm{CH}), 130.83(\mathrm{C}=\mathrm{C} \text {, aliphatic } \\
\text { carbon), } 122.54\left(\mathrm{CF}_{3}\right), 141.08-115.36 \text { (aromatic carbons), } \\
23.40\left(\mathrm{CH}_{3}\right)\end{array}$ \\
\hline $5 d$ & $\begin{array}{c}3060(\mathrm{CH}), 1688 \\
(\mathrm{C}=\mathrm{O}), 1591(\mathrm{C}=\mathrm{C})\end{array}$ & $\begin{array}{c}7.73(\mathrm{~s}, 1 \mathrm{H}, \mathrm{CH}), 7.19-7.71(\mathrm{comp}, 8 \mathrm{H}, \mathrm{Ar}-\mathrm{H}) \\
3.51(\mathrm{~s}, 1 \mathrm{H}, \mathrm{CH}), 2.52(\mathrm{~s}, 1 \mathrm{H}, \mathrm{NH}), 1.24(\mathrm{~s}, 3 \mathrm{H}, \\
\left.\mathrm{CH}_{3}\right)\end{array}$ & $\begin{array}{c}162.50(\mathrm{C}=\mathrm{O}), 134.82(\mathrm{CH}), 130.82(\mathrm{C}=\mathrm{C} \text {, aliphatic } \\
\text { carbon), } 123.13\left(\mathrm{CF}_{3}\right), 139.82-112.12 \text { (aromatic carbons), } \\
21.73\left(\mathrm{CH}_{3}\right)\end{array}$ \\
\hline $5 e$ & $\begin{array}{c}3069(\mathrm{CH}), 1678 \\
(\mathrm{C}=\mathrm{O}), 1590(\mathrm{C}=\mathrm{C})\end{array}$ & $\begin{array}{c}7.62(\mathrm{~s}, 1 \mathrm{H}, \mathrm{CH}), 7.38-7.27 \text { (comp, } 8 \mathrm{H}, \mathrm{Ar}-\mathrm{H}) \\
3.40(\mathrm{~s}, 1 \mathrm{H}, \mathrm{CH}), 2.14(\mathrm{~s}, 1 \mathrm{H}, \mathrm{NH}), 1.25(\mathrm{~s} \\
\left.3 \mathrm{H}, \mathrm{CH}_{3}\right)\end{array}$ & $\begin{array}{c}161.97(\mathrm{C}-\mathrm{F}), 161.54(\mathrm{C}=\mathrm{O}), 135.33(\mathrm{CH}), 136.40(\mathrm{C}=\mathrm{C}, \\
\text { aliphatic carbon), } 141.15-114.16(\text { aromatic carbons }), \\
23.10\left(\mathrm{CH}_{3}\right)\end{array}$ \\
\hline
\end{tabular}


TABLE 6: Spectral data of 2,2-dimethyl-5-(fluorinatedbenzylidene)-1,3-dioxane-4,6-diones (7a-e).

\begin{tabular}{|c|c|c|c|}
\hline Entry & IR $\left(\mathrm{cm}^{-1}\right)$ & ${ }^{1} \mathrm{H}$ NMR $(\delta \mathrm{ppm})$ & ${ }^{13} \mathrm{C}$ NMR $(\delta \mathrm{ppm})$ \\
\hline $7 \mathbf{a}$ & $\begin{array}{c}3083(\mathrm{CH}), 1630(\mathrm{C}=\mathrm{O}) \\
1554(\mathrm{C}=\mathrm{C} \text { str }), 1055(\mathrm{C}-\mathrm{O})\end{array}$ & $\begin{array}{c}8.97(\mathrm{~s}, 1 \mathrm{H}, \mathrm{CH}), 7.70(\mathrm{~d}, 2 \mathrm{H}, \mathrm{Ar}-\mathrm{H} \\
J=8.1 \mathrm{~Hz}), 7.64(\mathrm{~d}, 2 \mathrm{H}, \mathrm{Ar}-\mathrm{H}, J=8.1 \mathrm{~Hz}) \\
2.12\left(\mathrm{~s}, 6 \mathrm{H}, \mathrm{CH}_{3}\right)\end{array}$ & $\begin{array}{c}164.91(\mathrm{C}=\mathrm{O}), 160.42(\mathrm{C}-\mathrm{F}), 154.08(\mathrm{CH}), 123.74(\mathrm{C}=\mathrm{C}, \\
\text { aliphatic carbon), } 134.02-118.12(\text { aromatic carbons }) \\
105.18(\mathrm{O}-\mathrm{C}-\mathrm{O}), 26.16\left(\mathrm{CH}_{3}\right)\end{array}$ \\
\hline $7 \mathbf{b}$ & $\begin{array}{c}2915(\mathrm{CH}), 1682(\mathrm{C}=\mathrm{O}) \\
1577(\mathrm{C}=\mathrm{C} \text { str }), 1130(\mathrm{C}-\mathrm{O})\end{array}$ & $\begin{array}{c}8.42(\mathrm{~s}, 1 \mathrm{H}, \mathrm{CH}), 7.61(\mathrm{~d}, 2 \mathrm{H}, \mathrm{Ar}-\mathrm{H} \\
J=8.2 \mathrm{~Hz}), 7.32(\mathrm{~d}, 2 \mathrm{H}, \mathrm{Ar}-\mathrm{H}, J=8.0 \mathrm{~Hz}) \\
1.83\left(\mathrm{~s}, 6 \mathrm{H}, \mathrm{CH}_{3}\right)\end{array}$ & $\begin{array}{c}164.27(\mathrm{C}-\mathrm{O}), 165.46-165.30(\mathrm{C}=\mathrm{O}), 150.03(\mathrm{CH}), 124.18 \\
\left(\mathrm{C}=\mathrm{C} \text {, aliphatic carbon), } 122.42\left(\mathrm{CF}_{3}\right), 134.85-120.06\right. \\
\text { (aromatic carbons), 107.92 }(\mathrm{O}-\mathrm{C}-\mathrm{O}), 27.12-26.97\left(\mathrm{CH}_{3}\right)\end{array}$ \\
\hline 7c & $\begin{array}{c}3072(\mathrm{CH}), 1680(\mathrm{C}=\mathrm{O}) \\
1583(\mathrm{C}=\mathrm{C} \text { str }), 1145(\mathrm{C}-\mathrm{O})\end{array}$ & $\begin{array}{c}8.77(\mathrm{~s}, 1 \mathrm{H}, \mathrm{CH}), 7.88(\mathrm{~d}, 2 \mathrm{H}, \mathrm{Ar}-\mathrm{H} \\
J=7.9 \mathrm{~Hz}), 7.47(\mathrm{~d}, 2 \mathrm{H}, \mathrm{Ar}-\mathrm{H}, J=7.9 \mathrm{~Hz}) \\
1.85\left(\mathrm{~s}, 6 \mathrm{H}, \mathrm{CH}_{3}\right)\end{array}$ & $\begin{array}{l}167.40-167.30(\mathrm{C}=\mathrm{O}), 149.26(\mathrm{CH}), 126.15(\mathrm{C}=\mathrm{C}, \\
\text { aliphatic carbon }), 122.42\left(\mathrm{CF}_{3}\right), 135.05-121.93(\text { aromatic } \\
\text { carbons }), 106.94(\mathrm{O}-\mathrm{C}-\mathrm{O}), 27.72-27.64\left(\mathrm{CH}_{3}\right)\end{array}$ \\
\hline $7 d$ & $\begin{array}{c}3060(\mathrm{CH}), 1685(\mathrm{C}=\mathrm{O}) \\
1584(\mathrm{C}=\mathrm{C} \text { str }), 1095(\mathrm{C}-\mathrm{O})\end{array}$ & $\begin{array}{c}8.81(\mathrm{~s}, 1 \mathrm{H}, \mathrm{CH}), 7.80(\mathrm{~s}, 1 \mathrm{H}, \mathrm{Ar}-\mathrm{H}), 7.43(\mathrm{~d} \\
2 \mathrm{H}, \mathrm{Ar}-\mathrm{H}, J=6.5 \mathrm{~Hz}), 1.75\left(\mathrm{~s}, 6 \mathrm{H}, \mathrm{CH}_{3}\right)\end{array}$ & $\begin{array}{l}166.22-166.10(\mathrm{C}=\mathrm{O}), 149.29(\mathrm{CH}), 126.85(\mathrm{C}=\mathrm{C}, \\
\text { aliphatic carbon }), 123.67\left(\mathrm{CF}_{3}\right), 135.95-122.33(\text { aromatic } \\
\text { carbons), } 107.04(\mathrm{O}-\mathrm{C}-\mathrm{O}), 27.72-27.60\left(\mathrm{CH}_{3}\right)\end{array}$ \\
\hline $7 e$ & $\begin{array}{c}2979(\mathrm{CH}), 1680(\mathrm{C}=\mathrm{O}) \\
1590(\mathrm{C}=\mathrm{C} \text { str }), 1130(\mathrm{C}-\mathrm{O})\end{array}$ & $\begin{array}{c}8.37(\mathrm{~s}, 1 \mathrm{H}, \mathrm{CH}), 7.55(\mathrm{~s}, 1 \mathrm{H}, \mathrm{Ar}-\mathrm{H}), 7.17(\mathrm{~d} \\
2 \mathrm{H}, \mathrm{Ar}-\mathrm{H}, J=7.3 \mathrm{~Hz}), 1.70\left(\mathrm{~s}, 6 \mathrm{H}, \mathrm{CH}_{3}\right)\end{array}$ & $\begin{array}{c}\text { 165.40-165.30 }(\mathrm{C}=\mathrm{O}), 163.22(\mathrm{C}-\mathrm{F}), 146.91(\mathrm{CH}), 127.05 \\
(\mathrm{C}=\mathrm{C} \text {, aliphatic carbon), } 134.35-120.63(\text { aromatic } \\
\text { carbons), 106.97 (O-C-O), 26.50-26.42 }\left(\mathrm{CH}_{3}\right)\end{array}$ \\
\hline
\end{tabular}

IR spectra of compounds have been carried out at FET, MITS, Laxmangarh, Sikar, Rajasthan, India. The purity of compounds was checked on thin layers of silica gel in various nonaqueous solvent systems, for example, ethyl acetate: $n$-hexane $(1: 9)$. IR spectra were recorded in $\mathrm{KBr}$ on a PerkinElmer Infrared L1600300 Spectrum Two Li Ta spectrophotometer and ${ }^{1} \mathrm{H}$ NMR spectra were recorded on Bruker Avance II 400 NMR spectrometer using DMSO- $\mathrm{d}_{6}$ and $\mathrm{CDCl}_{3}$ as solvent and tetramethylsilane (TMS) as internal reference standard. The analgesic activity of synthesized compounds was carried out in Goenka College of Pharmacy, Department of Pharmacology, Lakshmangarh, Sikar, Rajasthan, India.

General Procedure for Extraction of Lemon Juice. Fresh lemon was cut by using knife and then pieces were pressed manually using domestic presser to extract juice. Then juice was then filtered through cotton/muslin cloth and then through filter paper to remove solid material and to get clear juice which was used as a catalyst.

General Procedure for the Preparation of $\mathbf{3 a}-\boldsymbol{e}, \mathbf{5 a}-\boldsymbol{e}$, and $7 \boldsymbol{a}-\boldsymbol{e}$. A mixture of fluorinated aromatic aldehyde $(1 \mathrm{mmol})$ and 2-thioxo-4-thiazolidinone/3-methyl-1-phenyl-2-pyrazolin-5-one/Meldrum's acid $(1 \mathrm{mmol})$ was taken in single neck round bottom flask and to this lemon juice $(2 \mathrm{~mL})$ was added as catalyst. The reaction mixture was stirred at room temperature for the appropriate time required for the completion of reaction given in Table 2. The progress of reaction was monitored by TLC using ethyl acetate : $n$-hexane $(1: 9)$ as eluent. After the completion of the reaction, mixture was poured onto crushed ice, and the solid product obtained was filtered and isolated in pure form with no need of further purification. For comparative studies, 3a was synthesized using various solvents and catalysts. Results of synthesis of $\mathbf{3 a}$ under different reaction conditions are given in Tables 1 and 2. The structures of the newly synthesized compounds are determined on the basis of their FTIR, ${ }^{1} \mathrm{H}$ NMR, ${ }^{19} \mathrm{~F}$ NMR, ${ }^{13} \mathrm{C} \mathrm{NMR}$, and mass spectral data.

\section{Analgesic Activity}

Few compounds have been screened for analgesic activity. The analgesic properties of the target compounds were tested using a model of central analgesia where the painful stimulus is represented by a hot plate heated to $56^{\circ} \mathrm{C}$. Seven groups of 6 mice, each having an average weight of 25-35 g, were taken for study. The animals were kept for a week before the experiment under standard laboratory environment, with access to water ad libitum. The experiment consisted in measuring the reaction to pain as the time (in seconds) between the moment when the animal was placed on the plate and the moment when it begins to lick its back paws in response to painful stimulus.

The animals were treated as follows.

Group 1: control group received $0.5 \%$ sodium CMC $(1 \mathrm{mg} / \mathrm{kg})$ I.P.

Group 2: nimesulide $5 \mathrm{mg} / \mathrm{kg}$ was administered I.P.

Group 3: the $\mathbf{3 a}$ in dose level of $50 \mathrm{mg} / \mathrm{kg}$ was administered I.P.

Group 4: the $\mathbf{3 b}$ in dose level of $50 \mathrm{mg} / \mathrm{kg}$ was administered I.P.

Group 5: the 3d in dose level of $50 \mathrm{mg} / \mathrm{kg}$ was administered I.P.

Group 6: the $\mathbf{5 b}$ in dose level of $50 \mathrm{mg} / \mathrm{kg}$ was administered I.P.

Group 7: the $\mathbf{5 d}$ in dose level of $50 \mathrm{mg} / \mathrm{kg}$ was administered I.P.

The time response of the animal to painful stimulus was evaluated at $0,30,60$, and 90 minutes interval after the administration of the tested substances. The recorded results were used to calculate for each group of animals the average response time to painful stimulus and the standard error. Statistical analysis (ANOVA followed by using Dunnett's test) was performed for analgesic activity to ascertain the significance of the exhibited activity. Compounds $\mathbf{3 b}, \mathbf{5 d}$, and $\mathbf{5 d}$ 
TABLE 7: Analgesic activity of the fluorinated 5-Substitutedbenzylidene derivatives.

\begin{tabular}{|c|c|c|c|c|}
\hline & $0 \mathrm{~min}$ & $30 \mathrm{~min}$ & $60 \mathrm{~min}$ & $90 \mathrm{~min}$ \\
\hline Control & $1.33 \pm 0.210$ & $1.66 \pm 0.210$ & $1.50 \pm 0.223$ & $1.83 \pm 0.307$ \\
\hline Standard Drug & $1.5 \pm 0.223$ & $\begin{array}{c}3.5 \pm 0.428^{* *} \\
\mathbf{5 3} \%\end{array}$ & $\begin{array}{c}7.83 \pm 0.477^{* *} \\
\mathbf{8 1} \%\end{array}$ & $\begin{array}{c}13.16 \pm 0.166^{* *} \\
\mathbf{8 6 \%}\end{array}$ \\
\hline $3 a$ & $1.16 \pm 0.166$ & $\begin{array}{c}2 \pm 0.258^{\mathrm{ns}} \\
17 \%\end{array}$ & $\begin{array}{c}2 \pm 0.258^{\mathrm{ns}} \\
25 \%\end{array}$ & $\begin{array}{c}2.56 \pm 0.210^{\mathrm{ns}} \\
27 \%\end{array}$ \\
\hline $3 b$ & $1.66 \pm 0.210$ & $\begin{array}{c}3.16 \pm 0.307^{* *} \\
\mathbf{4 8 \%}\end{array}$ & $\begin{array}{c}4.83 \pm 0.307^{* *} \\
\mathbf{6 9 \%}\end{array}$ & $\begin{array}{c}9.66 \pm 0.557^{* *} \\
\mathbf{8 1 \%}\end{array}$ \\
\hline $3 d$ & $1.66 \pm 0.166$ & $\begin{array}{c}1.83 \pm 0.307^{\mathrm{ns}} \\
\mathbf{9 \%}\end{array}$ & $\begin{array}{c}2 \pm 0.258^{\mathrm{ns}} \\
\mathbf{2 5 \%}\end{array}$ & $\begin{array}{c}2.56 \pm 0.210^{\mathrm{ns}} \\
\mathbf{2 7 \%}\end{array}$ \\
\hline $5 \mathbf{b}$ & $1.5 \pm 0.223$ & $\begin{array}{c}3.16 \pm 0.307^{* *} \\
\mathbf{4 8 \%}\end{array}$ & $\begin{array}{c}5.5 \pm 0.223^{* *} \\
73 \%\end{array}$ & $\begin{array}{c}10.83 \pm 0.600^{* *} \\
\mathbf{8 3} \%\end{array}$ \\
\hline $5 d$ & $1.66 \pm 0.210$ & $\begin{array}{c}3.0 \pm 0.0 .258^{* *} \\
\mathbf{4 5 \%}\end{array}$ & $\begin{array}{c}4.5 \pm 0.428^{* *} \\
67 \%\end{array}$ & $\begin{array}{c}8.52 \pm 0.670^{* *} \\
\mathbf{7 8 \%}\end{array}$ \\
\hline
\end{tabular}

All values mean \pm S.E.M values using 6 animals in each group.

Significant differences with respect to control group were evaluated by ANOVA, Dunnett's test.

${ }^{*} P<0.05,{ }^{* *} P<0.01,{ }^{* * *} P<0.001$, ns: nonsignificant.

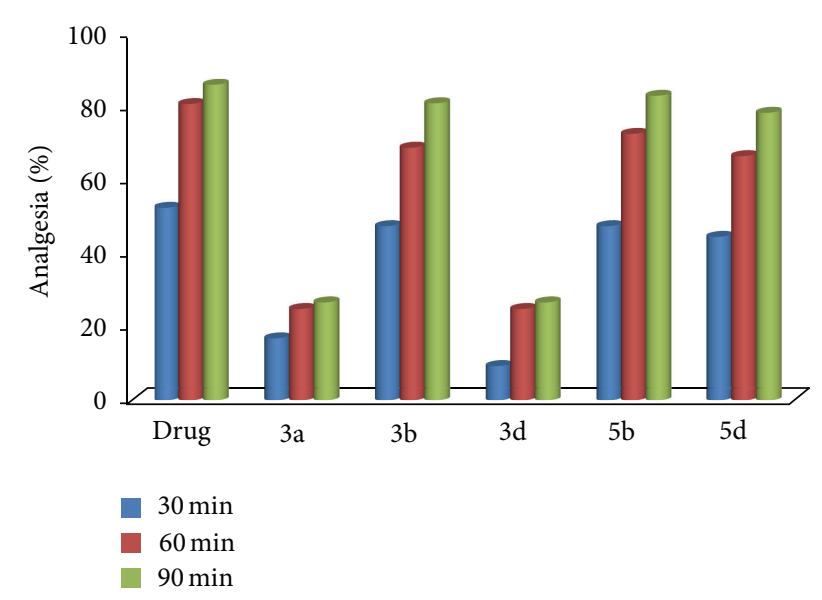

FIGURE 2: Comparison of the analgesic activity exhibited (\%) by the test and standard compounds at time interval of $30 \mathrm{~min}, 60 \mathrm{~min}$ and $90 \mathrm{~min}$.

have shown excellent analgesic activity as compared to other compounds which indicate that $\mathrm{OCF}_{3}$ group is more potent than $\mathrm{CF}_{3}$ and $\mathrm{NO}_{2}$ groups (Table 7, Figure 2).

\section{Conclusion}

The use lemon juice as green catalyst offers a convenient, nontoxic, inexpensive reaction medium for the synthesis of olefinic compounds. This procedure is simpler, economical, milder, and faster, including cleaner reactions, high yields of products and a simple experimental and work-up procedure, which makes it a useful and attractive process and is also consistent with the green chemistry theme which affords excellent yields. Compounds bearing $\mathrm{OCF}_{3}$ group possess excellent analgesic activity. With further molecular modification and manipulation of these compounds, several other promising bioactive molecules can be developed in future.

\section{Acknowledgments}

The authors are thankful to the Dean and HOD (Science and Humanities), FET, MITS, for providing necessary research facilities in the department. Financial assistance from FET, MITS is gratefully acknowledged. They are also thankful to SAIF, Punjab University, Chandigarh, India for the spectral analyses and Goenka College of Pharmacy, Department of Pharmacology, Lakshmangarh, Rajasthan, India for helping in performing analgesic activity.

\section{References}

[1] Ş. G. Küçükgüzel, S. Rollas, H. Erdeniz, M. Kiraz, A. Cevdet Ekinci, and A. Vidin, "Synthesis, characterization and pharmacological properties of some 4-arylhydrazono-2-pyrazoline-5one derivatives obtained from heterocyclic amines," European Journal of Medicinal Chemistry, vol. 35, no. 7-8, pp. 761-771, 2000.

[2] S. A. F. Rostom, I. M. El-Ashmawy, H. A. Abd El Razik, M. H. Badr, and H. M. A. Ashour, "Design and synthesis of some thiazolyl and thiadiazolyl derivatives of antipyrine as potential nonacidic anti-inflammatory, analgesic and antimicrobial agents," Bioorganic and Medicinal Chemistry, vol. 17, no. 2, pp. 882-895, 2009.

[3] S. Khode, V. Maddi, P. Aragade et al., "Synthesis and pharmacological evaluation of a novel series of 5-(substituted)aryl3-(3-coumarinyl)-1-phenyl-2-pyrazolines as novel anti-inflammatory and analgesic agents," European Journal of Medicinal Chemistry, vol. 44, no. 4, pp. 1682-1688, 2009.

[4] M. Abdel-Aziz, G. E. A. Abuo-Rahma, and A. A. Hassan, "Synthesis of novel pyrazole derivatives and evaluation of their antidepressant and anticonvulsant activities," European Journal of Medicinal Chemistry, vol. 44, no. 9, pp. 3480-3487, 2009.

[5] Z. S. Quan, R. L. Li, and Y. Z. Ling, "Study of the relationship between structure and anticonvulsant activities of 5-substituted-1-butry-3-pyrazolidinones and their synthesis," Acta Pharmaceutica Sinica, vol. 27, no. 9, pp. 711-716, 1992. 
[6] N. Das, A. Verma, P. K. Shrivastava, and S. K. Shrivastava, "Synthesis and biological evaluation of some new aryl pyrazol3-one derivatives as potential hypoglycemic agents," Indian Journal of Chemistry B, vol. 47, no. 10, pp. 1555-1558, 2008.

[7] G. A. Idrees, O. M. Aly, G. E. A. A. Abuo-Rahma, and M. F. Radwan, "Design, synthesis and hypolipidemic activity of novel 2-(naphthalen-2-yloxy)propionic acid derivatives as desmethyl fibrate analogs," European Journal of Medicinal Chemistry, vol. 44, no. 10, pp. 3973-3980, 2009.

[8] G. Ouyang, Z. Chen, X. Cai et al., "Synthesis and antiviral activity of novel pyrazole derivatives containing oxime esters group," Bioorganic and Medicinal Chemistry, vol. 16, no. 22, pp. 9699-9707, 2008.

[9] D. Castagnolo, F. Manetti, M. Radi et al., "Synthesis, biological evaluation, and SAR study of novel pyrazole analogues as inhibitors of Mycobacterium tuberculosis: part 2. Synthesis of rigid pyrazolones," Bioorganic and Medicinal Chemistry, vol. 17, no. 15 , pp. 5716-5721, 2009.

[10] K. B. Umesha, K. M. L. Rai, and M. A. Harish Nayaka, "Antioxidant and antimicrobial activity of 5-methyl-2-(5-methyl1,3-diphenyl-1H-pyrazole-4-carbonyl)-2,4-dihydro-pyrazol-3one," International Journal of Biomedical Science, vol. 5, no. 4, pp. 359-368, 2009.

[11] R. Tripathy, A. Ghose, J. Singh et al., "1,2,3-Thiadiazole substituted pyrazolones as potent KDR/VEGFR-2 kinase inhibitors," Bioorganic and Medicinal Chemistry Letters, vol. 17, no. 6, pp. 1793-1798, 2007.

[12] H. Park, K. Lee, S. Park et al., "Identification of antitumor activity of pyrazole oxime ethers," Bioorganic and Medicinal Chemistry Letters, vol. 15, no. 13, pp. 3307-3312, 2005.

[13] R. Murugan, S. Anbazhagan, and S. S. Narayanan, "Synthesis and in vivo antidiabetic activity of novel dispiropyrrolidines through $[3+2]$ cycloaddition reactions with thiazolidinedione and rhodanine derivatives," European Journal of Medicinal Chemistry, vol. 44, no. 8, pp. 3272-3279, 2009.

[14] S. Chandrappa, C. V. Kavitha, M. S. Shahabuddin et al., "Synthesis of 2-(5-((5-(4-chlorophenyl)furan-2-yl)methylene)-4-oxo2-thioxothiazolidin-3-yl)acetic acid derivatives and evaluation of their cytotoxicity and induction of apoptosis in human leukemia cells," Bioorganic and Medicinal Chemistry, vol. 17, no. 6, pp. 2576-2584, 2009.

[15] E. W. Brooke, S. G. Davies, A. W. Mulvaney et al., "Synthesis and in vitro evaluation of novel small molecule inhibitors of bacterial arylamine N-acetyltransferases (NATs)," Bioorganic and Medicinal Chemistry Letters, vol. 13, no. 15, pp. 2527-2530, 2003.

[16] S. Ozkirimli, F. Kazan, and Y. Tunali, "Synthesis, antibacterial and antifungal activities of 3-(1,2,4-triazol-3-yl)-4-thiazolidinones," Journal of Enzyme Inhibition and Medicinal Chemistry, vol. 24, no. 2, pp. 447-452, 2009.

[17] S. Chandrappa, S. B. Benaka Prasad, K. Vinaya, C. S. Ananda Kumar, N. R. Thimmegowda, and K. S. Rangappa, "Synthesis and in vitro antiproliferative activity against human cancer cell lines of novel 5-(4-methyl-benzylidene)-thiazolidine-2,4diones," Investigational New Drugs, vol. 26, no. 5, pp. 437-444, 2008.

[18] A. Verma and S. K. Saraf, "4-Thiazolidinone-a biologically active scaffold," European Journal of Medicinal Chemistry, vol. 43, no. 5, pp. 897-905, 2008.

[19] D. Davidson and S. A. Bernhard, “The structure of Meldrum's supposed $\beta$-lactonic acid," Journal of the American Chemical Society, vol. 70, no. 10, pp. 3426-3428, 1948.
[20] K. Byun, Y. Mo, and J. Gao, "New insight on the origin of the unusual acidity of Meldrum's acid from $\alpha \beta$-initio and combined QM/MM simulation study," Journal of the American Chemical Society, vol. 123, no. 17, pp. 3974-3979, 2001.

[21] B. Chen, "Meldrum's acid in organic synthesis," Heterocycles, vol. 32, no. 3, pp. 529-597, 1991.

[22] L. F. Tietze and U. Beifuss, "The knoevenagel reaction," in Comprehensive Organic Synthesis, vol. 2, pp. 341-394, 1991.

[23] B. Pita, E. Sotelo, M. Suárez et al., "Pyridazine derivatives. Part 21: synthesis and structural study of novel 4-aryl-2,5-dioxo-8phenylpyrido[2,3-d]pyridazines," Tetrahedron, vol. 56, no. 16, pp. 2473-2479, 2000.

[24] F. C. Brown, C. K. Bradsher, and S. M. Bond, "Some 5-substituted rhodanines," Industrial \& Engineering Chemistry, vol. 45, pp. 1030-1032, 1953.

[25] K. Ramkumar, V. N. Yarovenko, A. S. Nikitina et al., "Design, synthesis and structure-activity studies of rhodanine derivatives as HIV-1 integrase inhibitors," Molecules, vol. 15, no. 6, pp. 3958$3992,2010$.

[26] J. Iwao and K. J. Tomino, "Synthesis of pyrazolo [3, 4-b] pyridine by knovengel condensation," Pharmaceutical Society of Japan, vol. 76, pp. 748-755, 1956.

[27] B. A. Alekseenko, T. E. Gorizdra, and S. N. Baranov, "Synthesis and structure of noncondensed bicyclic thiazolidino-4-one derivatives," Khimiya Geterotsiklicheskikh Soedinenii, vol. 5, pp. 230-231, 1969.

[28] G. G. Allan, D. Maclean, and G. T. Newbold, "Condensation products of rhodanine, and keto-acids," Journal of the Chemical Society, pp. 5132-5153, 1952.

[29] F. C. Brown, C. K. Bradsher, S. G. McCallum, and M. Potter, "Rhodanine derivatives of ketones," Journal of Organic Chemistry, vol. 15, no. 1, pp. 174-176, 1950.

[30] M. M. Chowdhry, D. M. P. Mingos, A. J. P. White, and D. J. Williams, "Syntheses and characterization of 5-substituted hydantoins and thiazolines - Implications for crystal engineering of hydrogen bonded assemblies. Crystal structures t of 5-(2pyridylmethylene)-hydantoin, 5-(2-pyridylmethylene)-2-thiohydantoin, 5-(2-pyridylmethylene)thiazolidine-2,4-dione, 5-(2pyridylmethylene)rhodanine and 5-(2-pyridylmethylene)pseudothiohydantoin," Journal of the Chemical Society, Perkin Transactions 1, vol. 1, no. 20, pp. 3495-3504, 2000.

[31] R. V. Hangarge, D. V. Jarikote, and M. S. Shingare, "Knoevenagel condensation reactions in an ionic liquid," Green Chemistry, vol. 4, no. 3, pp. 266-268, 2002.

[32] S. S. Shindalkar, B. R. Madje, and M. S. Shingare, "Microwave induced, solvent-free Knoevenagel condensation of 4-oxo$(4 \mathrm{H})$-1-benzopyran-3-carbaldehyde with Meldrum's acid using alumina support," Indian Journal of Chemistry B, vol. 45, no. 11, pp. 2571-2573, 2006.

[33] S. Santosh, B. R. Shindalkar, R. V. Madje, P. T. Hangarge, M. K. D. Patil, and M. S. Shingare, "Borate zirconia mediated Knoevenagel condensation reaction in water," Journal of the Korean Chemical Society, vol. 49, pp. 377-380, 2005.

[34] S. S. Shindalkar, B. R. Madje, and M. S. Shingare, "Ultrasonically accelerated Knoevenagel condensation reaction at room temperature in distilled water," Indian Journal of Chemistry B, vol. 44, no. 7, pp. 1519-1521, 2005.

[35] N. B. Darvatkar, A. R. Deorukhkar, S. V. Bhilare, and M. M. Salunkhe, "Ionic liquid-mediated knoevenagel condensation of Meldrum's acid and aldehydes," Synthetic Communications, vol. 36, no. 20, pp. 3043-3051, 2006. 
[36] J. M. Khurana and K. Vij, "Nickel nanoparticles catalyzed chemoselective Knoevenagel condensation of Meldrum's acid and tandem enol lactonizations via cascade cyclization sequence," Tetrahedron Letters, vol. 52, no. 28, pp. 3666-3669, 2011.

[37] S. Ghosh, J. Das, and S. Chattopadhyay, "A novel light induced Knoevenagel condensation of Meldrum's acid with aromatic aldehydes in aqueous ethanol," Tetrahedron Letters, vol. 52, no. 22, pp. 2869-2872, 2011.

[38] A. M. Dumas, A. Seed, A. K. Zorzitto, and E. Fillion, "Triphenylphosphine mediated Knoevenagel condensation of Meldrum's acid with aromatic aldehydes," Tetrahedron Letters, vol. 48, pp. 7072-7276, 2007.

[39] K. Gong, Z. He, Y. Xu, D. Fang, and Z. Liu, "Green synthesis of 5-benzylidene rhodanine derivatives catalyzed by 1-butyl3-methyl imidazolium hydroxide in water," Monatshefte fur Chemie, vol. 139, no. 8, pp. 913-915, 2008.

[40] K. F. Shelke, S. B. Sapkal, B. R. Madja, B. B. Shingate, and M. S. Shingare, "Ionic liquid promoted an efficient synthesis of 5arylidene-2, 4-thiazolidinedione," Bulletin of the Catalysis Society of India, vol. 8, pp. 30-34, 2009.

[41] J. Zhou, Y. Song, F. Zhu, and Y. Zhu, "Facile synthesis of 5benzylidene rhodamine derivatives under microwave irradiation," Synthetic Communications, vol. 36, no. 22, pp. 3297-3303, 2006.

[42] K. Bourahla, A. Derdour, M. Rahmouni, F. Carreaux, and J. P. Bazureau, "A practical access to novel 2-amino-5-arylidene1,3-thiazol-4(5H)-ones via sulfur/nitrogen displacement under solvent-free microwave irradiation," Tetrahedron Letters, vol. 48, no. 33, pp. 5785-5789, 2007.

[43] S. Patil, S. D. Jadhav, and U. P. Patil, "Natural acid catalyzed synthesis of schiff base under solvent-free condition: as a green approach," Journal of Applied Sciences Research, vol. 4, no. 2, pp. 1074-1078, 2012.

[44] H. L. Yale, "The trifluoromethyl group in medicinal chemistry," Journal of Medicinal and Pharmaceutical Chemistry, vol. 1, no. 2, pp. 121-133, 1959.

[45] P. C. Appelbaum and P. A. Hunter, "The fluoroquinolone antibacterials: past, present and future perspectives," International Journal of Antimicrobial Agents, vol. 16, no. 1, pp. 5-15, 2000.

[46] F. M. D. Ismail, G. B. D. Michael, and J. Michael, "Modulation of drug pharmacokinetics and pharmacodynamics by fluorine substitution," Chemistry today, vol. 27, no. 3, pp. 18-21, 2009.

[47] H. Sachdeva, D. Dwivedi, K. Arya, S. Khaturia, and R. Saroj, "Anti-inflammatory activity, and QSAR study of some Schiff bases derived from 5-mercapto-3-(4'-pyridyl)-4H-1,2,4-triazol4-yl-thiosemicarbazide," Medicinal Chemistry Research, 2013.

[48] A. Dandia, H. Sachdeva, and R. Singh, "Improved synthesis of 3-spiro indolines in dry media under microwave irradiation," Synthetic Communications, vol. 31, no. 12, pp. 1879-1892, 2001.

[49] H. Sachdeva, D. Dwivedi, and S. Khaturia, "Aqua mediated facile synthesis of 2-(5/7-fluorinated-2-oxoindolin-3-ylidene)$\mathrm{N}$ - (4-substituted phenyl) hydrazine carbothioamides," Research Journal of Pharmaceutical, Biological and Chemical Sciences, vol. 2, no. 2, pp. 213-219, 2011.

[50] H. Sachdeva and D. Dwivedi, "Lithium-acetate-mediated biginelli one-pot multicomponent synthesis under solvent-free conditions and cytotoxic activity against the human lung Cancer Cell line A549 and Breast Cancer cell line MCF7," The Scientific World Journal, vol. 2012, Article ID 109432, 9 pages, 2012. 

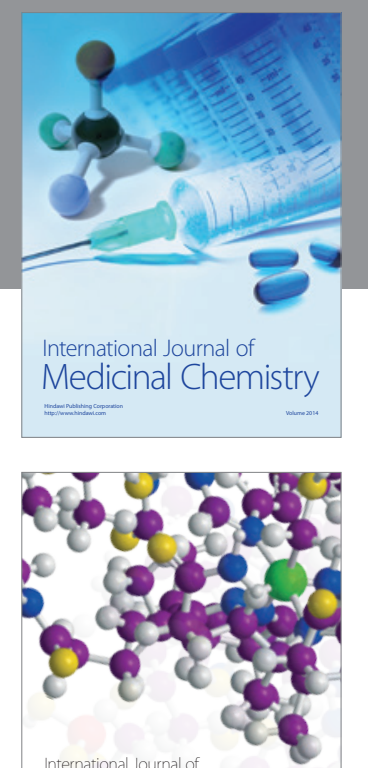

\section{Carbohydrate} Chemistry

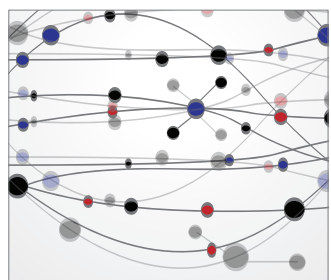

The Scientific World Journal
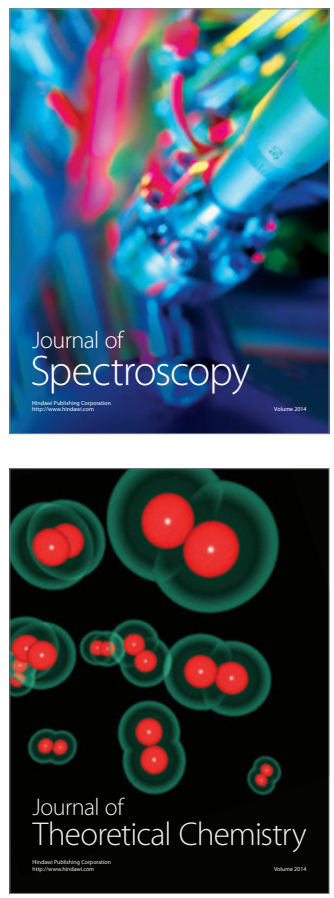
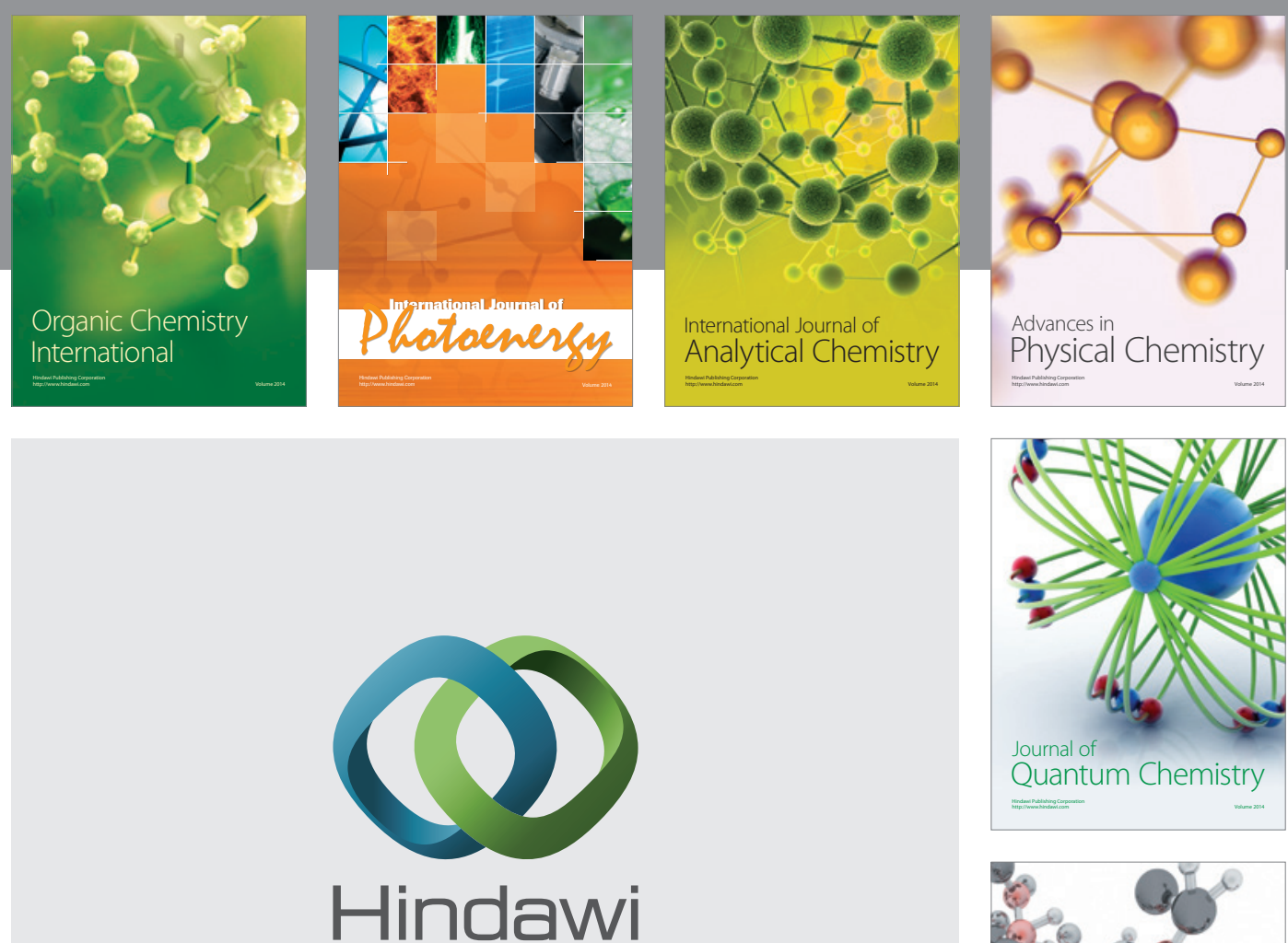

Submit your manuscripts at

http://www.hindawi.com

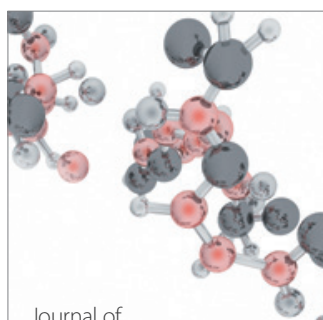

Analytical Methods

in Chemistry

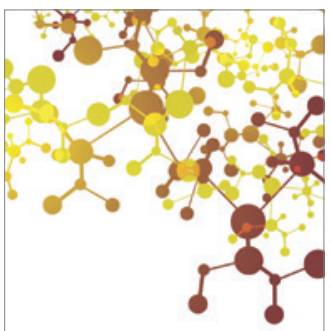

Journal of

Applied Chemistry

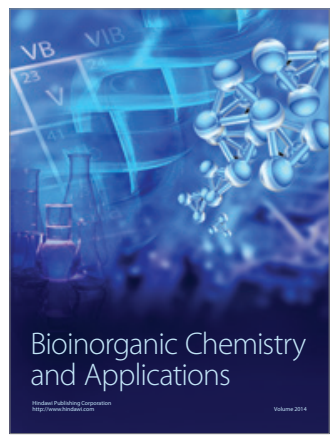

Inorganic Chemistry
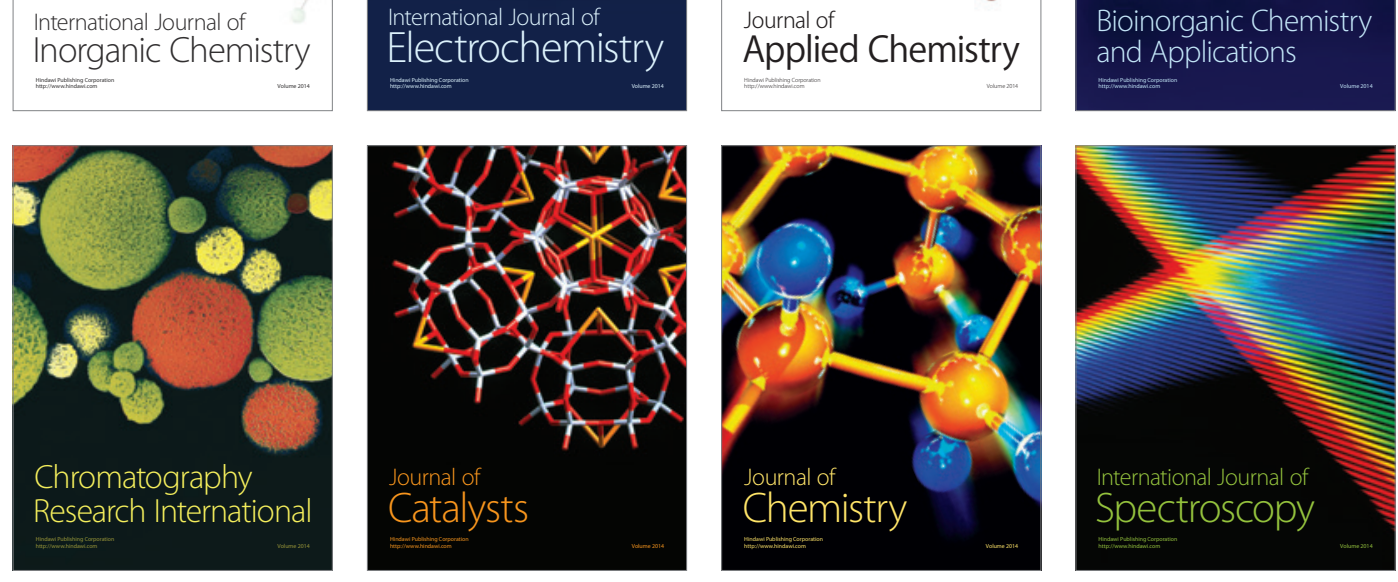